\title{
Nuevos horizontes en el desarrollo de medios de contraste en Radiología
}

\section{New horizons in the development of contrast media in Radiology}

\section{Vivas}

Servicio de Radiología.

Clínica Universidad de Navarra.

Pamplona
El poder diagnóstico de la radiología se basa en gran manera en la existencia de los medios de contraste (MC). Éstos son sustancias químicas que absorben fuertemente los rayos $\mathrm{X}$, de manera que las estructuras anatómicas que se tiñen con ellos son fácilmente visualizadas. En la mayoría de procedimientos diagnósticos los medios de contraste yodados se emplean por vía intravascular y contribuyen tanto a una mejor definición de la lesión como al estudio del comportamiento vascular de la misma ${ }^{1}$.

La historia de los MC comienza casi a la par que el descubrimiento de los rayos $\mathrm{X}$ en 1895. La mala visualización de los tejidos blandos en las primeras imágenes radiológicas hizo que científicos como Dutto, Hascheck y Lindenthal inyectaran algunas sutancias radiopacas como "yeso de París" o "pasta de Teichman" en vasos de cadáveres. En 1910 comenzó a utilizarse el bario para estudios gastrointestinales y en 1927 se realizó la primera angiografía carotídea con dióxido de torio. El yodo empezó a utilizarse en radiología con fines diagnósticos en 1929 por Moses Swick.

Los MC administrados de manera intravenosa se emplean sobre todo para la realización de angiografías y tomografías computarizadas (TC); tienen como molécula básica un anillo bencénico con tres átomos de yodo y se comercializan en forma de soluciones acuosas transparentes cuya concentración se expresa en $\mathrm{mg}$ de yodo/ml. Cuanto más yodo tenga el MC, mayor será su absorción de Rayos X y mayor será el grado de intensificación de los tejidos. En el momento actual en nuestro país, casi la totalidad de los medios de contraste que se emplean pertenecen al grupo de los conocidos como no iónicos. Éstos difieren de los iónicos en que no se disocian en el agua, por lo que su efecto diurético es inapreciable y su concentración es mayor en el sistema colector renal, lo que supone un ahorro en la dosis. Otra de sus características más relevantes es la mejor tolerancia por los pacientes, sin provocar apenas reacciones alérgicas ${ }^{2}$. 
Los MC radiológicos son administrados diariamente a miles de pacientes. Las mejoras fármaco-químicas logradas en las últimas décadas mejoran notablemente su seguridad y eficacia, pero no siempre son inocuos. Estos efectos, van a estar determinados por las características fisicoquímicas de los MC (hiperosmolaridad, viscosidad e hidrosolubilidad), así como las condiciones clínicas del paciente.

El artículo original de González Herrero y $\mathrm{col}^{3}$ que se publica en este número de Anales del Sistema Sanitario de Navarra estudia la incidencia de nefropatía inducida por contraste (NIC) en un grupo de pacientes diabéticos en tratamiento con metformina a los que se realiza un estudio de tomografía computarizada (TC) con contraste yodado (civ).

La nefropatía inducida por contraste yodado (NIC) se define como un empeoramiento de la función renal en los días inmediatamente posteriores a la administración intravascular de contraste en ausencia de otra causa de disfunción renal y tiene una incidencia que oscila entre el 0,6 al $50 \%{ }^{47}$, siendo la nefropatía diabética el factor de riesgo más importante para el desarrollo de NIC.

Cuando se realiza la inyección del MC durante una exploración radiológica, éste se diluye en la circulación y se difunde por el espacio intravascular, pasa a los capilares y de ahí al espacio intersticial o extracelular de los tejidos. Los MC no se metabolizan y una vez hacen su función diagnóstica se eliminan mayoritariamente a través del riñón por filtración glomerular, no existiendo excreción tubular ni reabsorción a nivel de los túbulos. Si la función renal es normal, más del $90 \%$ del MC se elimina en las primeras 24 horas. Sin embargo, si la función renal se encuentra comprometida (diabéticos, pacientes con insuficiencia renal, ancianos, hipertensos, pacientes con deshidratación, en tratamiento con furosemida, con enfermedad cardíaca, proteinuria e hipeuricemia) y el paciente toma metformina, el contraste se acumula más tiempo en el riñón, provocando toxicidad sobre los túbulos renales (oliguria a las $24-48 \mathrm{~h}$ de la exposición, o un incremento de los valores de creatinina en las 48-72 horas posteriores al procedimiento). No obstante, y como los autores aseveran en sus conclusiones $^{3}$, el riesgo actual de NIC en pacientes diabéticos a los que se administra contraste yodado es mínimo y solo en aquellos con insuficiencia renal sería recomendable retirar la metformina para evitar la acidosis láctica.

Además de los efectos tóxicos comentados, los MC pueden provocar efectos por hipersensibilidad de diferente entidad, aunque las reacciones adversas graves que requieren hospitalización o que ponen en riesgo la vida del paciente son muy $\operatorname{raras}^{4-7}$. La muerte es tan infrecuente que es muy difícil atribuirla en forma directa o exclusiva al medio de contraste. Casi siempre constituye una injuria más en pacientes con una enfermedad terminal, en general, en ancianos. La muerte en individuos jóvenes y sanos por shock anafiláctico es realmente excepcional. Las muertes comunicadas en la grandes series oscilan entre $1 / 100.000$ a $1 / 1000.000$.

El paciente, no obstante, tiene derecho a conocer todos los riesgos. De hecho la legislación vigente Ley básica 41/2002 reguladora de la Autonomía del paciente y de derechos y obligaciones en materia de información y documentación clínica (ley de autonomía del paciente) 
establece el derecho del paciente a la información relacionada con cualquier procedimiento al que sea sometido y especialmente con cualquier riesgo inherente al mismo.

También la SERAM (Sociedad Española de Radiología, www. seram.es) ha establecido una serie de recomendaciones al respecto del uso de los medios de contraste. Por una parte, los servicios de Radiología tienen la obligación de suministrar al paciente información escrita sobre la técnica, las posibles complicaciones y los riesgos, información que tiene una función complementaria a la verbal suministrada por el clínico. Por otra parte, el radiólogo deberá examinar, antes de la introducción del medio de contraste, los posibles factores de riesgo del paciente y en función del resultado, actuar en consecuencia.

Ante un paciente al que se solicita una prueba de imagen en el proceso diagnóstico, es responsabilidad del radiólogo valorar los pros y los contras de la administración de MC y conocer muy bien los efectos secundarios para prevenirlos cuando fuese posible y tratarlos adecuadamente cuando se desencadenasen.

$\mathrm{Al}$ igual que las mejoras fármaco-químicas logradas en las últimas décadas en pro de la seguridad y eficacia de los MC, en la actualidad se dedican muchos esfuerzos en evitar los efectos secundarios y en descubrir nuevas moléculas más precisas. Un grupo de investigadores de la Universidad de Salamanca y dos empresas biotecnológicas han ensayado en animales un método que mejora los efectos de la nefropatía de contraste a partir de la administración de la proteína denominada Cardiotrofina-1. El estudio, que se ha publicado en la revista Toxicological Sciences ${ }^{8}$, también ha revelado una parte de los mecanismos celulares y moleculares que explican esa protección y actualmente ya tiene aplicaciones clínicas directas.

Entre las estrategias más actuales y que en un futuro próximo estarán disponibles como medios de contraste para resonacia magnética, se encuentran las nanopartículas inyectadas de manera intravenosa. Se trata de nanoesferas de óxido de hierro de muy pequeño tamaño que presentan en su superficie pequeños péptidos o anticuerpos que pueden unirse a moléculas presentes en la superfice de las células tumorales ${ }^{9}$. La inyección intravenosa de pequeñas cantidades de estas nanopartículas permitiría una visualización más nítida de la lesión tumoral e incluso podría constituir una estrategia terapéutica no quirúrgica muy prometedora basada en la termoterapia. Esta estrategia ha sido ya implementada en un ensayo clínico en pacientes con tumores cerebrales malignos y ha abierto las puertas a futuros ensayos traslacionales en ésta y otras patologías neoplásicas. En la actualidad se está desarrollando una nueva generación de nanopartículas de sílice que pueden encapsular diferentes sustancias radiodensas (para TC) o radiactivas (para PET) ${ }^{10}$. Estas partículas radiodensas pueden combinarse con péptidos y anticuerpos con una gran capacidad de unión a la superficie de las células tumorales, confiriendo a estos compuestos una mayor sensibilidad para detectar la carga tumoral, una menor toxicidad como medio de contraste y un mejor perfil de eliminación renal. Esperamos que estas líneas de desarrollo, constituyan un avance en el radiodiagnóstico y en el desarrollo de nuevas herramientas terapéuticas. 


\section{BIBLIOGRAFÍA}

1. Méndez R, Graña L. Fármacos en Radiología. En: del Cura JL, Pedraza S, Gayete A, editores. Radiología Esencial. Madrid: Panamericana. 2009: p.65-77.

2. Martí-Bonmatí L, Pallardó Y. Monografía SERAM- Medios de contraste en radiología. Madrid: Editorial Médica Panamericana 2008.

3. González Herrero H, De Arriba Villamor C, Buldain Parra M, Arraiza Sarasa M. Nefrotoxicidad por contrastes yodados en estudios de tomografía computarizada a pacientes ambulatorios diabéticos en tratamiento con metformina. An Sist Sanit Navar 2013; 36: 197-201.

4. Katayama H, Yamaguchi K, Kozuka T, Takashima T, Seez P, Matsuura K. Adverse reactions to ionic and nonionic contrast media. A report from the Japanese Committee on the Safety of Contrast Media. Radiology 1990; 175: 621-628.

5. Lieberman P, Nicklas RA, Oppenheimer J, Kemp SF, Lang DM, Bernstein DI et al. The diagnosis and management of anaphylaxis practice parameter: 2012 Update. J Allergy Clin Immunol 2010; 126: 477-480.

6. WAO position paper. World Allergy Organization Guidelines for the Assessment and Manegement of Anphilaxys. WAO journal 2011; 4: 13-53.

7. Thomsen HS, Morcos SK. Management of acute adverse reactions to contrast media. Eur Radiol 2004; 14: 476-478.

8. Quiros Y, Sánchez-González PD, López-Hernández FJ, Morales AI, López-Novoa JM. Cardiotrophin-1 administration prevents the renal toxicity of iodinated contrast media in rats. Toxicol Sci 2013, 132: 493-501.

9. Wankhede, MW, Bouras A, Kaluzova M, HadjiPAnaYIS CG. Magnetic nanoparticles: an emergin technology for malignant brain tumor imagin and therapy. Expert Rev Clin Pharmacol. 2012; 5: 173-186.

10. Benezra M, Penate-Medina O, Zanzonico PB, Schaer D, Ow H, Burns A. Multimodal silica nanoparticles are effective cancer-targeted probes in a model of human melanoma. J Clin Invest 2011, 121: 2768-2780. 